\title{
Città, welfare e servizi: temi e questioni per il progetto urbanistico e le politiche sociali
}

Massimo Bricocoli, Stefania Sabatinelli

Politiche per progetti e una prospettiva di governance hanno aperto a forme interessanti di innovazione delle politiche di welfare. D'altra parte, numerosi sono le ambiguità e $i$ rischi sottesi in questa evoluzione sul piano della platea dei destinatari e dell'efficacia dei processi di inclusione sociale. Il saggio discute elementi di interesse e questioni che emergono nel progetto Welfare di Tutti a Milano mettendo in evidenza: a) potenzialità e prospettive per una riflessione che leghi progetto urbanistico e disegno delle politiche di welfare e organizzazione dei servizi, b) rischi e criticità di una declinazione territoriale del welfare locale in termini di aumento delle diseguaglianze e condizioni per l'inclusività del welfare

Parole chiave: welfare locale; piano dei servizi; piano di zona

City, Welfare and Services: Themes and Issues for Urban Planning and Social Policies

A project-led orientation in the design of public policies and an increasing orientation towards an extended governance of welfare has led to interesting innovations. Yet, many ambiguities and risks arise from this evolution in terms of the range of recipients and of the effectiveness of social inclusion measures. The article discusses questions and issues that emerge from the Welfare di Tutti project in Milan, with a focus on: a) potentials and perspectives for the integration of urban policies with the design and organization of social policies and services, $b$ ) risks and criticalities that emerge from the territorialisation of local welfare in terms of increasing inequalities and social inclusiveness of local welfare systems Keywords: social services; urban policies; local welfare
Politecnico di Milano, Dipartimento di Architettura e Studi Urbani (massimo.bricocoli@polimi.it; stefania.sabatinelli@polimi.it)

Il progetto dei servizi di welfare e la città: temi e prospettive. Politiche di welfare per progetti In modo estensivo, in molti campi dell'azione pubblica, il disegno di politiche avviene oggi soprattutto 'per progetti', ovvero nella forma di un palinsesto nel quale una molteplicità di attori concertano, secondo una geometria variabile, un insieme di progetti che nel loro insieme costituiscono parte consistente delle politiche (Dente et al., 1990). Come abbiamo visto, questo è certamente il caso del progetto Welfare di tutti e del suo ruolo rispetto all'attuazione del Piano di sviluppo delle politiche sociali della città di Milano.

La modalità di costruzione di politiche 'per progetti' è stata ampiamente investigata quale tratto che caratterizza il passaggio da un sistema rigido e gerarchico a un reticolo flessibile in cui si affermano e sviluppano progetti fondati sull'iniziativa e l'autonomia e che di volta in volta aggregano costellazioni di attori variabili (Boltanski, Chiappello, 1999; Bricocoli, Savoldi, 2012). Sviluppare politiche per progetti consente di definire un ambito di azione circoscritto, di mettere in relazione la filosofia di un approccio politico con un contesto e con dati reali e concreti, di accrescere la prossimità tra chi elabora le politiche e i destinatari anche in una direzione di maggiore partecipazione al processo decisionale. Ma un approccio per progetti appare più funzionale anche alle attese di valutazione degli effetti delle politiche (la loro efficienza, la loro efficacia) entro la matrice di new public management che sempre di più ha caratterizzato i modi di governo delle amministrazioni pubbliche e marcato la presa di distanza rispetto alle presunte forme di inefficienze, sprechi, sviluppi inerziali nel finanziamento dei programmi sociali. Un approccio per progetti si sviluppa entro orizzonti temporali definiti e quindi garantisce anche visibilità e riconoscimento all'azione di governo entro i tempi dei mandati politici. E ancora, tale approccio appare quanto mai aderente a un assetto di governance in cui il soggetto pubblico non è più necessariamente erogatore di politiche e servizi: l'iniziativa su politiche e progetti - e spesso anche la messa a disposizione delle risorse economiche - può fare capo a soggetti diversi (si pensi ad esempio al ruolo delle fondazioni e di Fondazione Cariplo in questo specifico caso).

Spinte e cambiamenti che più in generale possiamo ascrivere ad una logica di politiche per progetti e a un assetto di governance hanno aperto certamente a prospettive interessanti di innovazione e maggiore appropriatezza delle politiche di welfare. D'altra parte, numerosi sono le ambiguità e i rischi 
sottesi in questa evoluzione sul piano della platea effettiva dei destinatari e dell'efficacia dei processi di inclusione sociale, in un contesto in cui sono manifeste la fragilità dei sistemi di welfare locale e l'indebolimento delle protezioni sociali in generale (de Leonardis, 2016).

\section{Welfare di tutti 2015-2017: prime riflessioni}

Sebbene sia presto per definire quale sarà l'esito e la portata del progetto Welfare di Tutti nel lungo periodo, evidenziamo qui alcune prime riflessioni sui caratteri salienti che offrono elementi di interesse in una prospettiva di ricerca.

È senz'altro da rilevare l'intensa esperienza di cooperazione e concertazione tra l'amministrazione comunale e gli attori della governance territoriale nel costituire piattaforme fisiche e digital per l'erogazione dei servizi. In merito all'organizzazione dei servizi, la collaborazione e la presenza congiunta nelle attività di co-progettazione di operatori di diversi enti (pubblici e privati, diversi per settore, specializzazione, profilo) hanno aperto alla possibilità di una relazione più dinamica in luogo di una logica che in passato nel contesto milanese si è spesso configurata come mera esternalizzazione. La sperimentazione degli spazi WeMi quali nuovi luoghi di accesso ai servizi di welfare vede un'esposizione e un orientamento più proattivo dei servizi sociali che letteralmente guadagnano un affaccio su strada, quasi a marcare un riposizionamento rispetto alle postazioni più ordinarie - $\mathrm{e}$ meno accessibili - negli uffici.

Gli spazi WeMi, non si configurano esclusivamente come vetrine dei servizi domiciliari, ma sono concepiti quali veri e propri luoghi di incontro e di interazione leggera con i servizi sociali, dove si possano esplicitare bisogni, costruire relazioni entro una prospettiva volta a comporre nuove soluzioni e nuovi servizi. Lo spazio WeMi San Gottardo, in gran parte grazie all'attività del café e all'intraprendenza della cooperativa sociale che lo gestisce, ha già mosso i primi passi in questo senso, diventando sede di molte iniziative organizzate da diversi gruppi di cittadini e si è rivelato uno spazio accogliente e bello, significativo per gli operatori sociali, spinti a riconsiderare il proprio ruolo nei territori. In questo caso specifico, come abbiamo visto, la creazione di uno spazio 'ibrido' e non già 'polifunzionale' ha richiesto una procedura assai complessa e quindi costituito un precedente nel diritto urbanistico laddove l'ibridazione di due servizi (esercizio pubblico commerciale e servizi pubblici alla persona) ha richiesto un'inedita procedura di convenzionamento urbanistico (sia pure a una scala assai minuta!) che rappresenta oggi un riferimento importante nella giurisprudenza dell'amministrazione pubblica. Analoga complessità si è rivelata sul fronte del design della comunicazione, laddove era da affermare e valorizzare il connubio d'identità e di funzioni fra un café di iniziativa privata e un servizio sociale di iniziativa pubblica, entro il frame di un finanziamento da parte di una fondazione bancaria.

\section{Prospettive del progetto urbanistico:}

\section{la città dei servizi non è più/mai la stessa}

L'esperienza di coinvolgimento diretto nel progetto Welfare di Tutti ha consentito di misurarsi con un fronte di cambiamento che sollecita un riposizionamento di posture e pratiche disciplinari. Entro i tre anni di sviluppo del programma, il progetto dei servizi, il progetto urbanistico e architettonico, il design della comunicazione sono stati chiamati a giocare il proprio senso e rilevanza in modi assai inediti. La condizione di una regia pubblica capace e orientata all'innovazione e la partecipazione attiva a un processo che ha visto il coinvolgimento di una moltitudine di attori del terzo settore hanno consentito di mettere a fuoco le nuove istanze che la riorganizzazione dei servizi di welfare pone al progetto. Avendo sempre sullo sfondo le condizioni alle quali si danno oggi politiche e risorse per il welfare locale, le esplorazioni condotte entro un processo di intensa co-progettazione, l'attività di analisi e progetto alla scala ampia territoriale dei municipi e quartieri della città come a quella minuta di una cortina urbana e dello spaccato assonometrico di un locale con affaccio su strada, hanno consentito di avvicinare in modo concreto temi e questioni che sollecitano una diversa postura del progetto urbanistico rispetto al disegno e all'organizzazione dei servizi di welfare.

Qualità ed efficacia del welfare locale appaiono fortemente connesse alla capacità di attivazione e di intrapresa da parte di soggetti terzi. Nel disegno delle politiche di welfare locale assumono oggi particolare rilievo il contesto urbano/territoriale e la società locale, intesi insieme nella loro dimensione di specifica combinazione di risorse disponibili e ricomposte a livello locale per la promozione di progetti radicati nel territorio in grado in qualche modo di autosostenersi pur in assenza di trasferimenti economici costanti (Lo Piccolo, 2012; de Leonardis, 2016). L'innovazione sociale ha spesso tratti di specificità locale ed è negoziata tra attori e istituzioni che hanno una forte affiliazione e radicamento territoriale (Moulaert, 2009; Evers, 2014). La definizione di un diverso approccio alla progettazione e all'attivazione degli spazi dei servizi di welfare avviene sempre più laddove sono disponibili, insieme, risorse spaziali e intrapresa sociale. La rilevanza del contesto territoriale in senso materiale e concreto deriva altresì dal fatto che le risorse in campo cui attingere nella produzione di idee e per la realizzazione di progetti non sono appunto finanziarie, e dunque mobili, ma sono piuttosto risorse materiali che si danno - o meno - in uno specifico territorio. È il caso di quel tal immobile dismesso che potrebbe essere riconvertito, di alloggi sfitti in un determinato quartiere che potrebbero essere rimessi in gioco nel quadro di un progetto di inserimenti lavorativi, della cura di uno spazio collettivo o della coltivazione di orti urbani quali palestre di un nuovo protagonismo civico. E allora è fondamentale evidenziare il cambiamento di prospettiva che deriva dal mutamento strutturale del sistema di attori che producono i servizi e che implica innanzitutto un drastico cambiamento delle logiche di localizzazione. Se in una fase di espansione (urbana e del welfare) la pianificazione dei servizi è stata centrata sulla realizzazione di dotazioni pubbliche da distribuire (secondo una logica di infrastrutturazione per l'urbanistica e di bacino d'utenza per le politiche sociali), l'attivazione di servizi oggi avviene laddove si libera uno spazio, laddove sia possibile riutilizzare immobili e spazi già esistenti, non necessariamente di proprietà pubblica, da parte di una molteplicità di attori (pubblici, privati e non profit) che si muovono in modo fluido e spesso disancorato da pianificazione e programmazione.

La mappa - dei servizi - che ne risulta è quindi una mappa assai diversa da quella che fin qui il sapere urbanistico ha prodotto. E' una mappa in cui la presenza e la dislocazione dei servizi posso- 


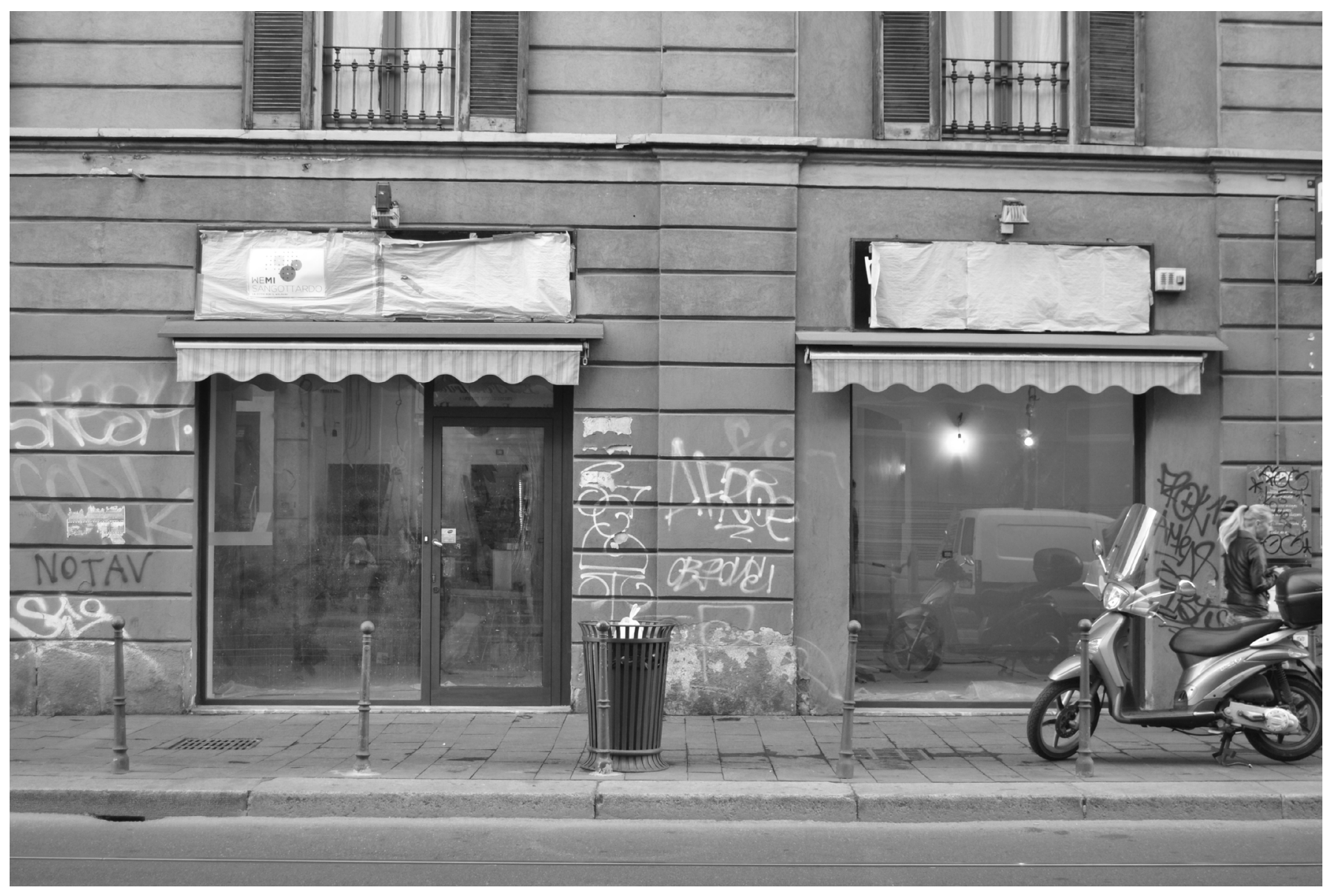

Vista esterna dello spazio Rab-Wemi durante l'esecuzione dei lavori

Fonte: archivio dell'autore, 2016 
no variare in un tempo relativamente breve, in corrispondenza dell'attivazione/disattivazione di iniziative, progetti, servizi (che siano residenziali, di assistenza, di integrazione o di cura...) e che dipendono da risorse spesso discontinue (spazi in usufrutto, usi temporanei, finanziamenti di progetti...). Questo comporta un deciso cambiamento di prospettiva. Si tratta di procedere a un ripensamento dei modi in cui l'urbanistica guarda e orienta la configurazione spaziale dei servizi sia tenendo in maggior conto la dimensione temporale, sia aprendo ad una maggiore estensione delle scale di intervento. La rilevanza di una scala ampia nella pianificazione dei servizi permane quale condizione per orientare equità e razionalità nella distribuzione dei servizi alla scala urbana. Ma è nel progetto e nella regolazione dello spazio alla scala architettonica (ovvero ad una scala minuta che ancora fatica a essere assunta come parte rilevante del progetto urbanistico) che si gioca molto del potenziale di accessibilità, inclusività, efficacia, generatività e attrattività degli spazi dei servizi di welfare.

A valle dell'esperienza del progetto WeMi e in una prospettiva di generalizzazione dei risultati conseguiti e di apprendimento istituzionale, appare importante promuovere e alimentare una maggiore integrazione tra il disegno di politiche e pratiche in ambito urbanistico e quelle nel campo delle politiche sociali. Con riferimento al caso lombardo e alla strumentazione normativa in uso, un fronte sul quale appare fondamentale investire maggiori energie, sia in termini di ricerca che di supporto esperto ai processi decisionali, è quello dei piani sfalsati sui quali si muovono il disegno e la costruzione del piano dei servizi in ambito urbanistico e la definizione del piano di zona o piano di sviluppo del welfare. Colpisce, nel caso del Comune di Milano come nella generalità dei casi in Lombardia, l'assenza di integrazione e connessioni tra strumenti di pianificazione che, a partire dalla loro titolazione, auspicheremmo in stretta relazione. Il Comune di Milano si appresta nel 2017 ad aggiornare il Piano dei Servizi che accompagna il Piano di Governo del Territorio. Nello stesso periodo saranno avviate le procedure per disegnare il nuovo Piano di Zona che definisce le linee strategiche di sviluppo del welfare locale. Al di là delle retoriche, la sperimentazione del progetto WeMi costituisce un riferimento importante per mettere concretamente alla prova la possibilità di integrare due fronti di pianificazione che fino ad ora hanno operato in modo disgiunto e affatto sinergico. L'estensione e la replicabilità di un progetto innovativo nel campo delle politiche sociali risulta in buona parte legata alla capacità della pianificazione urbanistica di recepirne ed elaborarne le sollecitazioni, sia per indirizzare nuove politiche sulla base di una gamma di bisogni fortemente differenziata, sia per dotare la città di nuovi e diversi spazi in cui il welfare locale sia presente e accessibile.

\section{Declinazioni territoriali del welfare locale e diseguaglianze in città}

Se mai c'è stata una tendenza all'universalismo, come tensione e aspirazione di politiche che miravano a un'allocazione di risorse materiali e di protezioni sociali (Granaglia, 2016), a meno di una salda regia pubblica, esiste in prospettiva un rischio importante che l'articolazione territoriale del welfare locale alimenti la crescita di sperequazioni e squilibri, fino all'esclusione dall'accesso ai servizi. Si tratta di una tendenza che possiamo collocare entro uno sfondo, ben descritto da Jacques Donzelot (2009), entro il quale la forza della cittadinanza sociale prodotta dal welfare state nel secolo scorso (e sovrascritta alle condizioni di cittadinanza civile e politica conquistate nei secoli precedenti) appare oggi sempre più messa a rischio a fronte della crisi dello stato sociale (Lo Piccolo, 2012). Ad avanzare è un'idea di 'cittadinanza urbana' che si declina sempre più in chiave territoriale, contestualizzata e quindi per forza di cose più discrezionale $\mathrm{e}$ diseguale laddove alcune regioni, aree, quartieri urbani sono avvantaggiate nell'accesso ai servizi (e, quindi, ai diritti) e altre invece risultano penalizzate.

In questo processo di progressiva disarticolazione delle dotazioni e della distribuzione delle misure di welfare, la città stessa, i suoi municipi, i suoi quartieri, rischiano di essere elementi divisivi piuttosto che unificanti, laddove viene rimessa in campo un'immagine premoderna in cui la protezione sociale era esplicitamente garantita da forme di appartenenza/adesione comunitaria (Donzelot, 2009; Ernesti, 2011; Bianchetti, 2014, 2016). Tale processo ha dunque tratti fortemente controversi e contraddittori. Nelle situazioni urbane di maggiore crisi corrisponde certamente a soluzioni che cercano di compensare l'assenza di servizi di welfare e attrezzature pubbliche (Lo Piccolo, 2012; Laino, 2012). In altre condizioni - ed è questo il caso milanese - rientra in una prospettiva di governance in cui si definiscono e costruiscono alleanze tra soggetti pubblici e terzo settore. In una prospettiva di ricerca e di progettazione all'intersezione tra politiche urbane e politiche sociali, ci interessa qui mettere in evidenza alcuni rischi e criticità che una declinazione territoriale del welfare locale ha in termini di aumento delle diseguaglianze e delle condizioni per l'inclusività del welfare.

Più specificatamente, quattro diversi fattori emergono con evidenza. Un primo fattore deriva da cambiamenti profondi del sistema di protezioni sociali a livello nazionale e si gioca dunque, prima ancora che al livello della città/locale, in corrispondenza dei modi e delle forme di allocazione delle risorse agli individui. Tra gli altri, il processo di devoluzione che ha esacerbato l'autonomia regionale, ha comportato disparità di trattamento dei cittadini nei diversi territori.

Un secondo fattore di rischio risulta accentuato alla scala urbana, laddove emerge con evidenza un orientamento a configurare politiche e servizi che si ancorano ad una logica territoriale, locale e che trova ampia adesione nei progetti e nelle retoriche del 'welfare di comunità' che lega in varia misura l'accesso ai servizi al radicamento, all'appartenenza ad una entità territorialmente definita. È questo un terreno in cui nel sistema di governance locale del welfare, politiche e progetti sembrano sempre più promuovere e alimentare quell'idea di 'cittadinanza urbana' che abbiamo richiamato in apertura. Il caso delle politiche abitative e dell'orientamento dei progetti di housing sociale e abitare condiviso è emblematico. Come abbiamo avuto modo di discutere (Bricocoli, de Leonardis, 2014), le forme di protezione sociale si ridisegnano a ridosso dei singoli, in corrispondenza di progetti che mobilitano dispositivi selettivi e un'impronta comunitaria nella modalità di assegnazione e gestione delle abitazioni.

Infine, un quarto fattore deriva, insieme, dall'assetto di governance e dalle implicazioni che ne discendono in termini di geografia dei servizi. Come abbiamo visto, l'attivazione e la dislocazione di servizi nella città segue logiche che non sono necessariamente sovraimposte ma che dipendono fortemente 
dall'iniziativa di soggetti terzi (privati e non profit), dagli spazi che sono nella loro disponibilità, dalle reti e dal radicamento nei diversi contesti che ne ispirano e orientano le scelte. La localizzazione di risorse e strutture è oggi esito quasi casuale del riuso di risorse storicamente deputate ad altre funzioni, e acquisite agli interventi di welfare per vie diverse, come la dismissione e riconversione di grandi strutture militari, le donazioni di patrimoni individuali, per definizione dispersi sul territorio, a enti benefici o pubblici, la confisca di beni alle mafie, ecc. A titolo di esempio: nel 2015, nell'ambito del laboratorio Housing and Neighbourhoods centrato sui temi del welfare locale, gli studenti del corso di Laurea magistrale in Urban Planning and Policy Design hanno realizzato un lavoro di mappatura in un Municipio della città di Milano che ha messo in evidenza come la più parte dei servizi alla persona si sia nel tempo dislocata lungo un asse collocato ad ovest della zona, lasciando ampiamente 'scoperta' un'ampia e popolosa porzione della zona ad est. Un'analisi più attenta metteva in evidenza che è lungo quel primo asse che sono localizzate le sedi delle più importanti imprese del terzo settore attive nella zona.

Nella pluralità di attori che operano in campo sociale, una delle funzioni fondamentali in capo all'attore pubblico consiste dunque nel saper ricomporre la mappa dell'esistente, a supporto della programmazione, della ricostruzione di filiere e percorsi dotati di senso e di una verifica complessiva degli effetti che gli assetti di governance hanno in termini di accessibilità complessiva alle risorse.

Si aggiunga a questo che in una prospettiva in cui attività, progetti e servizi in ambito sociale costituiscono una componente complementare e per alcuni versi subordinata rispetto ad altre attività (e risorse connesse), la disponibilità all'intrapresa vede in tensione una logica più inclusiva e propria dell'impresa sociale e una logica d'impresa più prettamente economica. Se questo è storicamente un tratto connaturato e insieme controverso dell'impresa sociale (de Leonardis et al., 1994) sono da considerare con grande attenzione gli effetti della crescente enfasi sulla sostenibilità economico-finanziaria dei progetti e delle attività che è riferimento guida per la più parte dei programmi di finanziamento. Questo è certamente un elemento che orienta e discrimina luoghi e quindi persone laddove è selettivo rispetto a parametri di efficienza, solvenza e reddittività, e non invece di priorità e intensità dei bisogni sociali.

\section{Riferimenti bibliografici}

Boltanski L., Chiappello E., 1999, Le nouvel esprit du capitalism. Paris: Gallimard (ed. it., 2014, Il nuovo spirito del capitalismo. Milano: Mimesis). Bianchetti C., 2014, a cura di, Territori della condivisione. Una nuova città. Macerata: Quodlibet.

Bifulco L., 2015, Il welfare locale. Processi e prospettive. Roma: Carocci. Bricocoli M., de Leonardis O., 2014, «Le protezioni sociali ravvicinate. Sogni e incubi». In: Bianchetti C. (a cura di), Territori della condivisione. Una nuova città. Macerata: Quodlibet.

de Leonardis O., Mauri D., Rotelli F., 1994, L'impresa sociale. Milano: Anabasi. de Leonardis O., 2016, a cura di, «I nodi dell'inclusione sociale e la riorganizzazione del welfare. La questione abitativa». In: Urban@it (a cura di), Rapporto sulle città. Bologna: il Mulino.

Dente B., Bobbio L., Fareri P., Morisi M., 1990, Metropoli per progetti. Attori e processi di trasformazione urbana a Firenze, Torino, Milano. Bologna: il Mulino.

Donzelot J., 2009, Vers une citoyenneité urbaine? La ville et l'égalité des chances. Paris: Editions Rue d'Ulm. Doi: 10.3917/ulm.donze.2009.01. Ernesti G., 2012, «Welfare, città, cultura del piano». In: Munarin S., Martelliano V., Spazi, Storie e soggetti del welfare. Roma: Gangemi.

Evers, A., Ewert, B., 2014, «Introduction. Social Innovations for Social Cohesion: 77 Cases from 20 European Cities». In: Evers A., Ewert B., Brandsen T. (eds.), Social innovations for social cohesion. www. wilcoproject.eu/book/chapters/social-innovations-for-social-cohesion-77-cases-from-20-european-cities/.

Granaglia E., 2016, «Farewell to Universalism? Some Introductory Remarks». Politiche sociali, 3: 391-402. Doi da verificare.

Kazepov Y., 2010, ed., Rescaling Social Policies Towards Multilevel Governance in Europe. Avebury: Ashgate.

Laino G., 2012, Il fuoco nel cuore e il diavolo in corpo. La partecipazione come attivazione sociale. Milano: FrancoAngeli.

Lo Piccolo F., 2012, «Welfare o diritto alla città? Sguardi e voci plurali nell'esercizio di forme di cittadinanza attiva». In: Munarin S., Martelliano V., Spazi, storie e soggetti del welfare. Roma: Gangemi.

Moulaert F., MacCallum D., Mehmood A., Hamdouch A., 2014, eds., The International Handbook on Social Innovation. Collective Action, Social Learning and Transdisciplinary Research. Edward Elgar. Doi: $10.4337 / 9781849809993$

Munarin S., Martelliano V., 2012, Spazi, storie e soggetti del welfare. Roma: Gangemi.

Petrella F., 2010, Occasioni di dialogo. Torino: Antigone.

Pierson P., 1998, «Irresistible Forces, Immovable Objects: Post-industrial Welfare States Confront Permanent Austerity». Journal of European Public Policy, 5, 4: 539-560. Doi: 10.1080/13501769880000011.

Sabatinelli S., 2016, «Aspetti critici dell'innovazione sociale nel contesto italiano». Prospettive Sociali e Sanitarie, 1: 8-11. 